\title{
Nuclear Matrix Elements for Neutrinoless Double Beta Decay from Lattice QCD
}

\author{
William Detmold \\ Center for Theoretical Physics, Massachusetts Institute of Technology, MA 02139, USA \\ E-mail: wdetmoldemit.edu \\ David Murphy* \\ Center for Theoretical Physics, Massachusetts Institute of Technology, MA 02139, USA \\ E-mail: djmurphy@mit.edu
}

\begin{abstract}
While neutrino oscillation experiments have demonstrated that neutrinos have small, nonzero masses, much remains unknown about their properties and decay modes. One potential decay mode - neutrinoless double beta decay $(0 \vee \beta \beta)$ - is a particularly interesting target of experimental searches, since its observation would imply that the neutrino is a Majorana particle, demonstrate that lepton number conservation is violated in nature, and give further constraints on the neutrino masses and mixing angles. Relating experimental constraints on $0 v \beta \beta$ decay rates to the neutrino masses, however, requires theoretical input in the form of non-perturbative nuclear matrix elements which remain difficult to calculate reliably. In this talk we will discuss progress towards first-principles calculations of relevant nuclear matrix elements using lattice QCD and effective field theory techniques, assuming neutrinoless double beta decay mediated by a light Majorana neutrino. We will show preliminary results for the $\pi^{-} \rightarrow \pi^{+} e^{-} e^{-}$transition amplitude computed on a $16^{3} \times 32$ domain wall fermion lattice with a pion mass of $420 \mathrm{MeV}$, and discuss improved methods applicable to general lattice calculations of $0 v \beta \beta$ decay amplitudes.
\end{abstract}

The 39th International Conference on High Energy Physics (ICHEP2018)

4-11 July, 2018

Seoul, Korea

${ }^{*}$ Speaker. 


\section{Introduction}

Neutrinoless double beta decay $(0 v \beta \beta)$, if observed, would provide a wealth of information about the properties of neutrinos, as well as an example of a process violating lepton number conservation in nature. Relating experimental measuremets of the $0 v \beta \beta$ decay rate for a particular nucleus to the effective Majorana neutrino mass $m_{\beta \beta}=\left|\sum_{k} U_{e k}^{2} m_{k}\right|$ requires theoretical input in the form of a nuclear matrix element $M^{0 v}$ describing the non-perturbative, hadronic part of the decay. Reliably calculating these matrix elements has proven to be a difficult challenge, with predictions for a given nucleus from different nuclear model calculations differing by $100 \%$ or more [1].

In principle, lattice QCD provides an entirely ab-initio method for determining $M^{0 v}$, however, the large nuclei relevant to $0 v \beta \beta$ searches are computationally intractable for the foreseeable future. More realistically, one could hope to compute matrix elements of quark-level processes such as $n n \rightarrow$ ppee, and relate these to matrix elements of many-body systems within an effective field theory framework, or else to study small nuclei and probe the systematics of nuclear model calculations by directly comparing lattice and model predictions. First calculations of the long-distance contributions to the neutrinoful double beta decay process $n n \rightarrow$ ppeev $v$ and of the short-distance contributions to neutrinoless double beta decay were reported in Refs. [2] and [3], respectively. In this work we discuss first steps toward computing the long-distance contributions to $0 v \beta \beta$.

\section{Methodology}

We assume throughout that neutrinoless double beta decay is mediated by the long-distance light Majorana neutrino exchange mechanism. At low energies the underlying Standard Model interaction responsible for this decay is described by the effective electroweak Hamiltonian $\mathscr{H}_{W}=$ $2 \sqrt{2} G_{F} V_{u d}\left(\bar{u}_{L} \gamma_{\mu} d_{L}\right)\left(\bar{e}_{L} \gamma_{\mu} v_{e L}\right) .0 v \beta \beta$ is induced at second order in electroweak perturbation theory, leading to the bilocal matrix element

$$
\int d^{4} x d^{4} y\left\langle f e e\left|T\left\{\mathscr{H}_{W}(x) \mathscr{H}_{W}(y)\right\}\right| i\right\rangle=4 m_{\beta \beta} G_{F}^{2} V_{u d}^{2} \int d^{4} x d^{4} y H_{\alpha \beta}(x, y) L_{\alpha \beta}(x, y),
$$

where $L_{\alpha \beta} \equiv \bar{e}_{L}\left(p_{1}\right) \gamma_{\alpha} S_{V}(x, y) \gamma_{\beta} e_{L}^{C}\left(p_{2}\right) e^{-i p_{1} \cdot x} e^{-i p_{2} \cdot y}$ and $H_{\alpha \beta} \equiv\left\langle f\left|T\left\{\bar{u}_{L}(x) \gamma_{\alpha} d_{L}(x) \bar{u}_{L}(y) \gamma_{\beta} d_{L}(y)\right\}\right| i\right\rangle$ are tensors describing the leptonic and hadronic parts of the decay, respectively, $S_{v}(x, y)$ is the neutrino propagator, and $e_{L}^{C} \equiv C \bar{e}_{L}^{\top}$ denotes charge conjugation. Lattice QCD allows for a fully non-perturbative treatment of the hadronic tensor with full control over systematics.

To develop methodology we begin by considering the simplest $0 v \beta \beta$ process: the pion transition $\pi^{-} \rightarrow \pi^{+} e^{-} e^{-}$. We extract the desired matrix element by employing methods which have been successfully applied to other second-order electroweak processes on the lattice, including the neutrinoful double beta decay process $n n \rightarrow$ ppeev $v$ [2] and kaon decays [4]. By inserting a sum over intermediate states $n$ into the bilocal matrix element of Eqn. (2.1) it can be shown that the analogous lattice correlation function has the asymptotic time dependence

$$
C_{\pi \rightarrow \pi e e}(t) \propto \sum_{n} \frac{\left|Z_{\pi}\right|^{2}}{4 m_{\pi}^{2}} \frac{e^{-m_{\pi} t}}{2 E_{n}} \frac{\left\langle\pi e e\left|\mathscr{H}_{W}\right| n\right\rangle\left\langle n\left|\mathscr{H}_{W}\right| \pi\right\rangle}{E_{n}-m_{\pi}}\left[T+\frac{e^{-\left(E_{n}-m_{\pi}\right) T}-1}{E_{n}-m_{\pi}}\right]
$$

for pions at rest, where $T$ is the size of the temporal integration window for the weak current insertions and $t=\left|t_{+}-t_{-}\right|$is the $\pi^{-}-\pi^{+}$source-sink separation. At large $T$ one can extract the 
matrix element $M^{0 v}=\sum_{n}\left\langle\pi e e\left|\mathscr{H}_{W}\right| n\right\rangle\left\langle n\left|\mathscr{H}_{W}\right| \pi\right\rangle /\left(E_{n}-m_{\pi}\right)$ from a linear fit to the $T$ dependence of Eqn. (2.2).

We expect the lowest energy intermediate states to consist of a purely leptonic state $\left|e \bar{v}_{e}\right\rangle$ and a single pion state $\left|\pi e \bar{v}_{e}\right\rangle$, which require special consideration. The $\left|e \bar{v}_{e}\right\rangle$ state contributes a term to Eqn. (2.2) which grows exponentially as $T \rightarrow \infty$, while, for the $\left|\pi e \bar{v}_{e}\right\rangle$ state, the energy denominator $E_{n}-m_{\pi} \approx m_{e}$ becomes small, potentially contributing a term $\propto T^{2}$. The remaining tower of multi-hadron states have energies $E_{n}>m_{\pi}$ and thus will contribute terms to Eqn. (2.2) which are asymptotically linear at large $T$.

\section{Pilot Lattice Study of the $\pi^{-} \rightarrow \pi^{+} e^{-} e^{-}$Decay}

We have performed a pilot calculation using 1000 independent gauge field configurations of a $16^{3} \times 32 \times 16$ domain wall fermion ensemble with a lattice cutoff of $a^{-1}=1.6 \mathrm{GeV}$, physical volume of $(2 \mathrm{fm})^{3}$, and an unphysically heavy quark mass corresponding to a $420 \mathrm{MeV}$ pion. We use Coulomb gauge-fixed wall source propagators for the quarks, and a free overlap propagator with an infinite temporal extent for the neutrino. To reduce the computational cost such that the calculation can be performed with current resources we follow the strategy employed in Ref. [4] and treat the weak current insertions asymmetrically: the operator at $x$ is fixed at the (spatial) origin while the operator at $y$ is integrated over the spatial directions. Improved methods which will be used in future lattice calculations are discussed in Section 4.

In the left panel of Figure 1 we plot the integrated bilocal matrix element described by Eqns. (2.1) and (2.2) as a function of $T$, with the overall factor of $4 m_{\beta \beta} G_{F}^{2} V_{u d}^{2}$ removed, for a wide range of neutrino masses $m_{e} / 3 \lesssim m_{\beta \beta} \lesssim 2 m_{\pi}$. For $m_{\beta \beta}<m_{\pi}$ we observe the expected exponential divergence at large $T$ from the $\left|e \bar{v}_{e}\right\rangle$ intermediate state, as well as the emergence of a consistent $m_{\beta \beta} \rightarrow 0$ limit. We conclude that our calculation is insensitive to the precise choice of $m_{\beta \beta}$ over the range of experimentally relevant neutrino masses. A preliminary analysis in which we have also computed the transition amplitude to the $\mid e \bar{v}_{e}$ intermediate state and used this to remove the exponentially growing term in Eqn. (2.2) is summarized in the right panel of Figure 1 and in Table 1.

\begin{tabular}{cccc}
\hline \hline$\left[\frac{\left\langle\pi e e\left|\mathscr{H}_{W}\right| n\right\rangle\left\langle n\left|\mathscr{H}_{W}\right| \pi\right\rangle}{E_{n}-m_{\pi}}\right] /\left[\sum_{n} \frac{\left\langle\pi e e\left|\mathscr{H}_{W}\right| n\right\rangle\left\langle n\left|\mathscr{H}_{W}\right| \pi\right\rangle}{E_{n}-m_{\pi}}\right]$ & $-0.0082(15)$ & $1.0082(13)$ & $0.00009(26)$ \\
\hline \hline
\end{tabular}

Table 1: Preliminary results for the relative contributions from the hadronic vacuum $(n=0)$, single pion $(n=1)$ and remaining higher energy intermediate states $(n \geq 2)$, to $M^{0 v}$.

\section{Exact Treatment of the Neutrino Propagator}

Lattice QCD calculations of many-body systems are known to suffer from signal-to-noise problems. In anticipation of future calculations with baryonic and nuclear initial and final states, where we expect such signal-to-noise problems to enter, we have explored methods for performing an exact integration of the matrix element (2.1) over the spacetime locations of both current insertions; naively one expects an $\mathscr{O}(1 / \sqrt{V})$ reduction in the statistical error compared to the single sum method of our pilot study. We use a Gaussian-regulated, infinite volume, continuum, scalar 

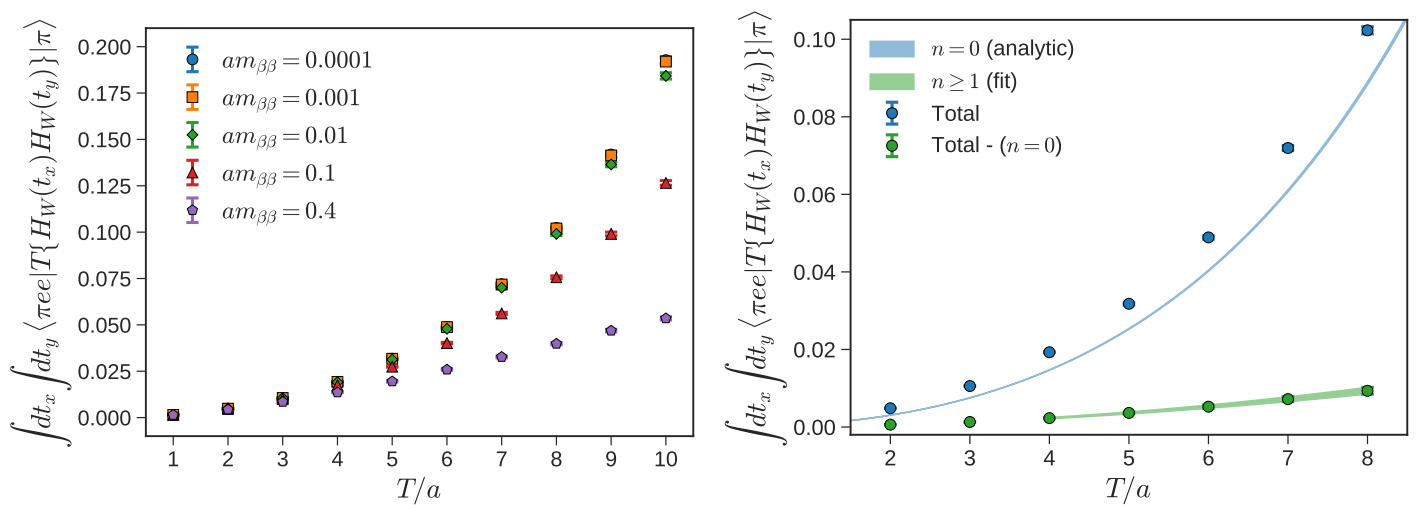

Figure 1: Left: Preliminary results for the integrated bilocal matrix element (Eqn. (2.1)) for a variety of neutrino masses given in lattice units. Right: Result for the lightest neutrino mass decomposed into the total, contribution from the $\left|e \bar{v}_{e}\right\rangle$ intermediate state, and the sum over contributions from the single pion and higher energy intermediate states.

propagator for the neutrino, which we expect to reduce finite volume effects compared to a lattice neutrino propagator, and exploit the convolution theorem and the fast Fourier transform to perform the full double integration in $\mathscr{O}(V \log V)$. For more detail we direct the interested reader to Ref. [5].

\section{Conclusions}

We have performed an exploratory lattice QCD calculation of the $\pi^{-} \rightarrow \pi^{+} e^{-} e^{-}$transition amplitude on a $16^{3} \times 32 \times 16$ domain wall fermion ensemble, and developed substantially improved methods applicable to general $0 v \beta \beta$ decay amplitudes. We are currently using these methods to compute the $\pi^{-} \rightarrow \pi^{+} e^{-} e^{-}$amplitude on $24^{3} \times 64 \times 16$ domain wall fermion ensembles at multiple pion masses, and including short-distance contributions [3] as well as the long-distance contributions described in this work. Analyzing these results and matching them to $\chi \mathrm{PT}$, as well as extending our calculations to include baryonic and nuclear initial and final states, will be the subject of future studies.

\section{References}

[1] A. Giuliani et al., "Neutrinoless Double-Beta Decay”, Adv. High Energy Phys. (2012) 857016.

[2] B. Tiburzi et al., "Double- $\beta$ Decay Matrix Elements from Lattice Quantum Chromodynamics", Phys. Rev. D 96 (2017) 054505.

[3] A. Nicholson et al., "Heavy Physics Contributions to Neutrinoless Double Beta Decay from QCD", Phys. Rev. Lett. 121, (2018) 172501.

[4] Z. Bai et al., " $K^{+} \rightarrow \pi^{+} v \bar{v}$ Decay Amplitude from Lattice QCD”, Phys. Rev. D 98 (2018) 074509.

[5] W. Detmold and D. Murphy, "Nuclear Matrix Elements for Neutrinoless Double Beta Decay from Lattice QCD", to appear in PoS Lattice2018 (2018) 262. 